\title{
Silica-coated metals and semiconductors. Stabilization and nanostructuring*
}

\author{
Marta Alejandro-Arellano1, Thearith Ung², Álvaro Blanco³, \\ Paul Mulvaney2, and Luis M. Liz-Marzán1 \\ ${ }^{1}$ Departamento de Química Física, Universidade de Vigo, E-36200, Vigo, Spain; \\ 2Nanotechnology Laboratory, School of Chemistry, University of Melbourne, 3512 \\ Parkville, Australia; ${ }^{3}$ Propiedades Ópticas, Magnéticas y de Transporte, Instituto de \\ Ciencia de Materiales de Madrid (CSIC), Campus de Cantoblanco, 28049, Madrid, \\ Spain
}

\begin{abstract}
We present in this paper the use of silica-coating for nanostructuring metal and semiconductor nanoparticles. The basic concept is the strict tailoring of the interparticle spacing through the thickness of the silica shell. Three different experiments are presented that exemplify this concept. The first example consists of the preparation of thin films using the layer-by-layer self-assembly of gold nanoparticles, either uncoated or coated with thin silica shells. The observed optical effects are interpreted using effective medium theory. The second and third experiments are related to the preparation of three-dimensional nanostructures, either as concentrated dispersions of thickly coated Au or CdS nanoparticles, or as opals prepared from such core-shell nanoparticles. Within these crystalline solids, intercore distance is again dictated by the thickness of the silica shells.
\end{abstract}

\section{INTRODUCTION}

One of the most important developments in chemical technologies is that of nanostructuring [1]. Nanostructured materials are assemblies of nano-sized units which display unique, characteristic properties at a macroscopic scale. The size range of such units lies within the colloidal range, where the individual properties are different to both those of atoms/molecules and to those of the bulk [2]. Therefore, the properties of the nanostructured assemblies can be tuned by varying the colloidal properties of the constituents, mainly particle size, surface properties, interparticle interactions, and interparticle distance.

In this paper we discuss a procedure that allows for both stabilization of different types of nanoparticles and nanostructuring in such a way that interparticle distance can be chosen at will. The basis for this double effect is the coating of each nanoparticle with a shell of an inert material $\left(\mathrm{SiO}_{2}\right)$, whose thickness can be controlled with nanometer precision. The unusual properties of silica, especially in aqueous media [3] provide the particles with a very much enhanced colloidal stability, so that concentrated dispersions can be prepared, and they remain stable over a much wider range of solution conditions, such as ionic strength, temperature, solvent polarity, etc. This means that the procedures for nanostructuring such colloidal units are more robust and reproducible. Additionally, the ability to control the thickness of the silica shell implies that the separation between neighboring particles can be tuned, so that the collective behavior of the particles within the nanostructure can be tailored.

We show here three different ways to prepare nanostructured systems, using silica-coated metal or semiconductor nanoparticles as units. First, the preparation of thin films using the layer-by-layer self-assembly of gold nanoparticles with varying interparticle separation is shown to lead to strong

*Pure Appl. Chem. 72, 1-331 (2000). An issue of reviews and research papers based on lectures presented at the $1^{\text {st }}$ IUPAC Workshop on Advanced Materials (WAM1), Hong Kong, July 1999, on the theme of nanostructured systems.. 
optical effects, which can be interpreted on the basis of effective medium theory. Then, we show the preparation of concentrated dispersions of thickly coated Au or CdS nanoparticles, as well as the optical effects derived from interparticle separation. Finally, we show the first opals prepared from such coreshell nanoparticles. Within these crystalline solids, intercore distance is again dictated by the thickness of the silica shells.

\section{SILICA COATING}

A few methods can be found in the literature for the coating of colloids of different nature with silica. Iler [4] pioneered this technique, using the deposition of silica from sodium silicate solutions of certain concentrations and pH, which he termed "active silica". In the early 1990s, Ohmori and Matijevic [5] used the hydrolysis of alkoxysilanes for coating iron oxides, and then Philipse's group in Utrecht used a combination of these two procedures for coating both magnetite [6] and boehmite rods [7]. However, even this combination fails with noble metals, given their reluctance to bind to metal oxides. A first way to cope with this vitreophobicity of metals was to attach gold nanoparticles to small silica host colloids,

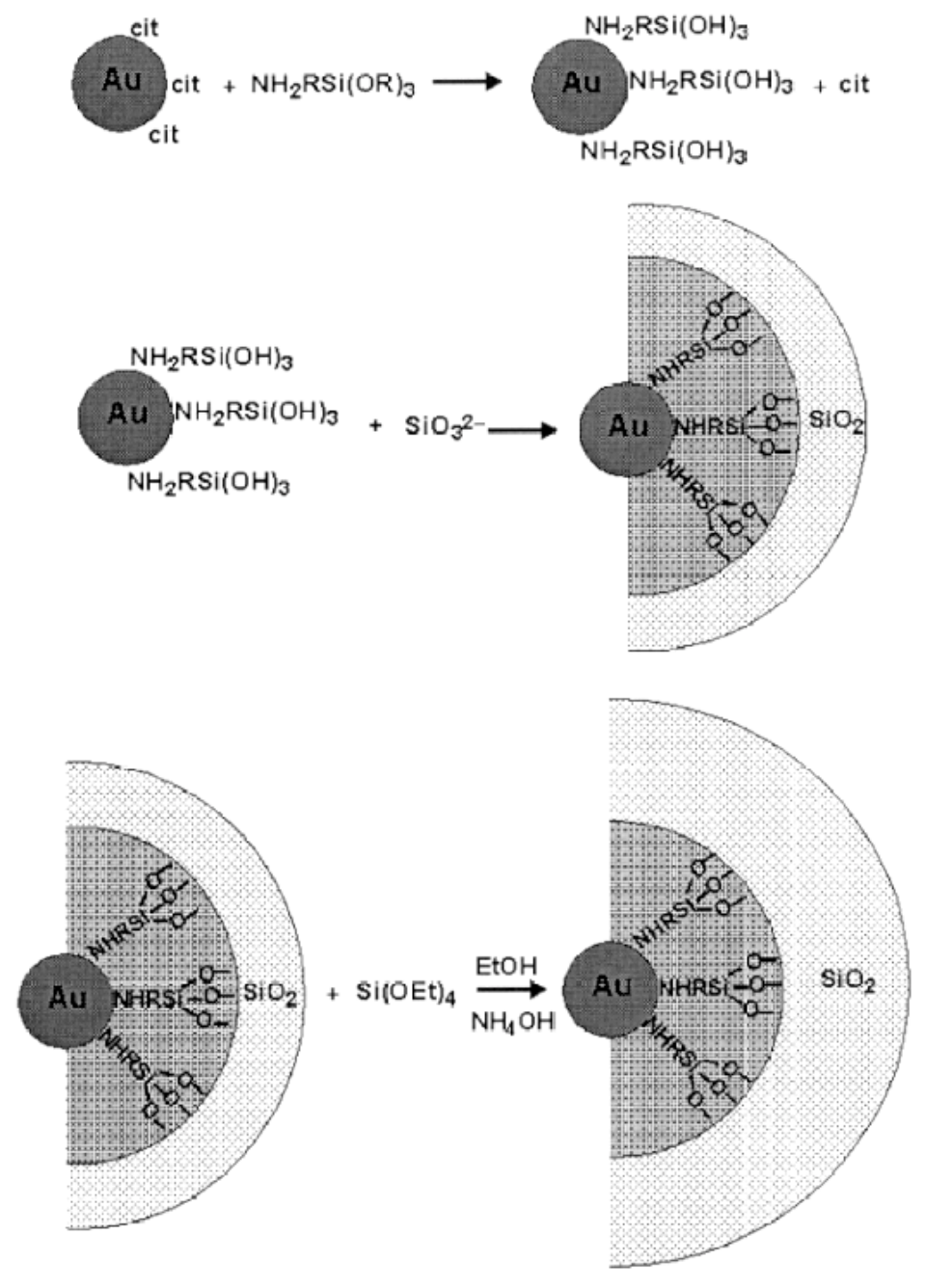

Fig. 1 Schematic representation of the silica-coating procedure. 
and then to grow the resulting composite particles in order to embed the Au as a core [8]. Though this method proved to be effective, a large population of (normally undesired) core-free silica particles was present as well. It should be mentioned that the use of microemulsions as nanoreactors for the controlled growth on the cores was used first by Asher for CdS [9], and very recently by Adair et al. for Ag [10].

We recently devised an alternative method [11,12], which uses silane coupling agents as surface primers to render the particle surface vitreophilic. The process is sketched in Fig. 1 for the case of gold. Silane coupling agents [13] are used as surface primers to provide the nanoparticle surface with silanol groups, and therefore to endow them with chemical affinity for silica, so that they can be coated by means of the procedures previously used for oxides. For metal nanoparticles, (3-aminopropyl) trimethoxysilane (APS) is used as a coupling agent, because of the large complexation constant for noble metal amines [14]. In the case of semiconductor particles like CdS, silane coupling agents with mercapto groups are preferred [15]. During this first step, the small organic ions initially adsorbed onto the surface are displaced by the coupling agents, which show a larger adsorption affinity. In a second step, active silica (as defined by Iler [4]) is added to the dispersion, which promotes the formation of a thin, dense, and relatively homogeneous silica layer around the particles [11], using the silanol groups as anchor points. At this stage, the particles can be transferred into ethanol, and the silica layer thickness can be increased in a controlled way by means of the base-catalyzed hydrolysis and condensation of tetraethoxysilane (TES), which is known as the Stöber process [16]. Figure 2 shows a high-resolution

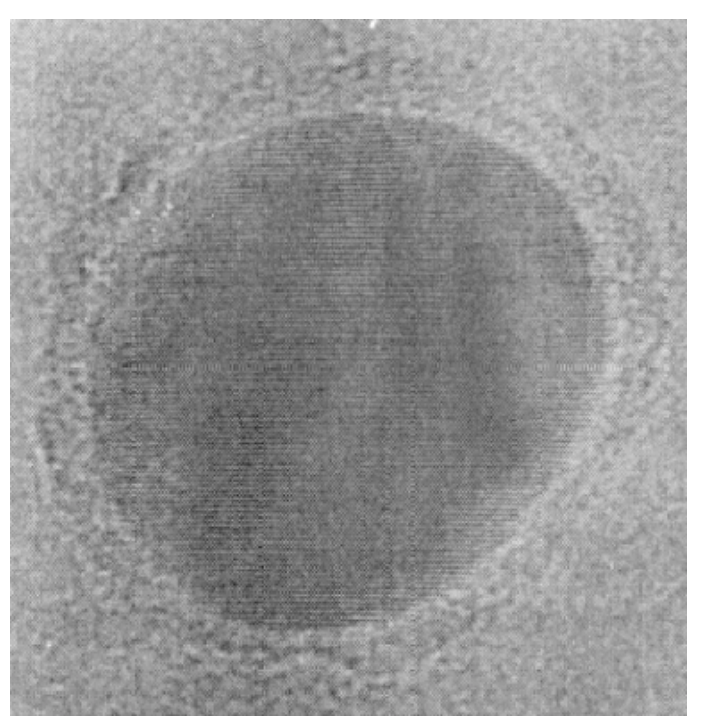

Fig. 2 High-resolution electron micrograph of a $10-\mathrm{nm} \mathrm{Ag}$ single crystal coated with an amorphous silica shell some $2 \mathrm{~nm}$ thick.

transmission electron micrograph of one $\mathrm{Ag} @ \mathrm{SiO}_{2}$ particle, illustrating the clear contrast between the metal core and the amorphous, thin silica shell.

\section{NANOSTRUCTURED THIN FILMS FROM AU@SiO}

We made use of the method of layer-by-layer self-assembly for the deposition of mono- or multilayers of gold nanoparticles. This method, initially developed for oppositely charged micron-sized colloids [17], was later successfully extended to polyelectrolyte pairs [18,19] and many other systems [20-22]. Nowadays, it is one of the most widely used for the preparation of nanoparticle-based thin films because of its versatility, universality, and ease of implementation. The method comprises the initial deposition 
of a layer of polyelectrolyte on a substrate, followed by deposition of oppositely charged nanoparticles by simple dipping of the modified substrate in the aqueous colloid. For our experiments, given the negative surface charge on silica surfaces above the iep, we made use of a positively charged polyelectrolyte (poly(diallyldimetylammonium) chloride or PDDA). For the build-up of thin films comprising more than one polyelectrolyte-nanoparticle layer, the dipping procedure is repeated until the desired number of layers is attained. The mono- and multilayers so prepared are very uniform, and the nanoparticles are in close contact with each other, so that interparticle distance will be determined through the thickness of the shell. By means of this procedure we have deposited thin films of $13-\mathrm{nm} \mathrm{Au}$ particles on standard microscope glass slides, with interparticle separations ranging between 0 and 30 $\mathrm{nm}$. We used two different sorts of samples for the "bare" gold films, one of them citrate stabilized and the second stabilized with 3-mercaptopropionic acid. These two samples, which are stabilized by organic molecules, display colors which are very similar to those of a film prepared on the same substrate by conventional sputtering. However, for larger interparticle separations, obtained by simple control of the deposited silica shell thickness, the color progressively changes until, for a separation of $30 \mathrm{~nm}$, the film has a very similar appearance to that of the starting aqueous sol.

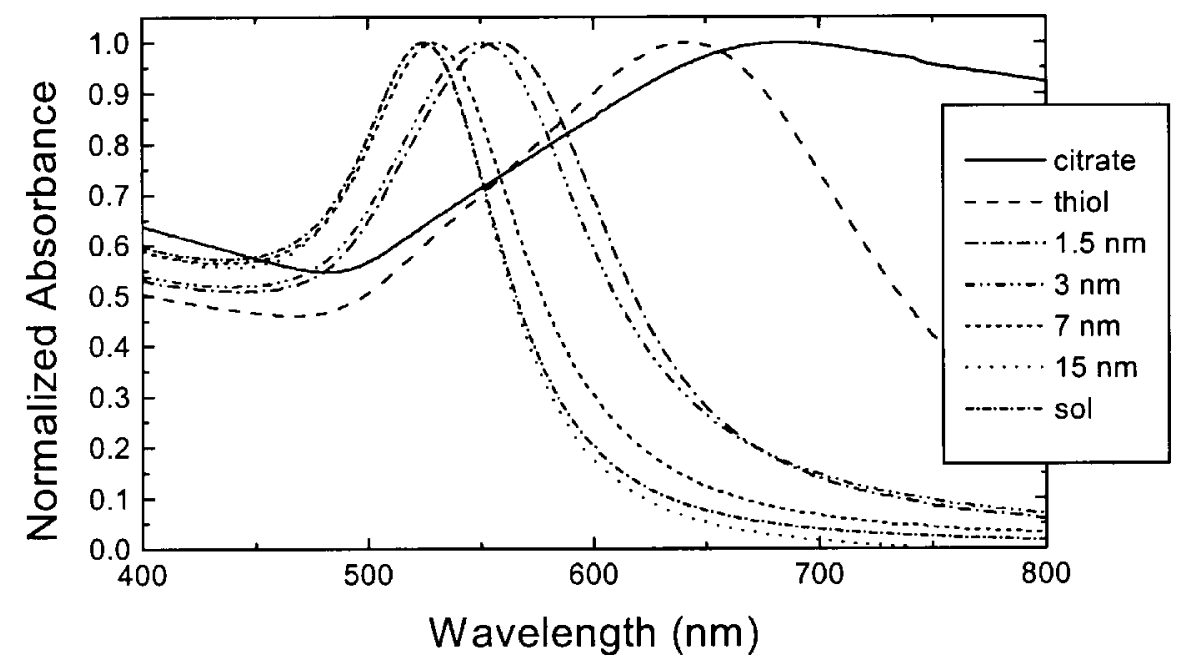

Fig. 3 Visible spectra of thin films prepared by successive deposition of 10 monolayers of Au nanoparticles coated with silica shells of increasing thickness.

A more quantitative way of observing this effect is through the corresponding visible absorption spectra. These are shown in Fig. 3, where we can see how the surface plasmon band blue-shifts as the interparticle separation is increased, until for $30 \mathrm{~nm}$ distance the spectrum basically coincides with that of the corresponding aqueous sol.

This effect can be explained by a decrease in dipole-dipole interactions between neighboring particles. As the separation increases, the influence of the oscillating dipoles of neighboring particles on the frequency of a central particle will decrease accordingly. There are numerous models to account for the dipole interaction between the gold colloid particles, which cause these color shifts. These "effective" medium models date back to Maxwell-Garnett, and Bruggemann [23,24]. The coupling is most simply treated using Maxwell-Garnett theory, which provides an expression for the effective dielectric constant of the composite material as, 


$$
\varepsilon_{\mathrm{eff}}=\varepsilon_{\mathrm{m}}\left(\frac{3+2 f \alpha}{3-\mathrm{f} \alpha}\right)
$$

where

$$
\alpha=3\left(\frac{\varepsilon-\varepsilon_{\mathrm{m}}}{\varepsilon+2 \varepsilon_{\mathrm{m}}}\right)
$$

$f$ and $\varepsilon$ are the volume fraction and the frequency-dependent complex dielectric constant of the metal particles respectively, and $\varepsilon_{\mathrm{m}}$ is the real dielectric constant of the surrounding medium. Equation 2 is valid when the particle radius and the interparticle separation are small compared with the wavelength of light, and when the volume fraction is moderately low. If the metal particles are surrounded by a concentric shell of material with a dielectric constant $\varepsilon_{\mathrm{s}}, \alpha$ is given by:

$$
\alpha=3\left(\frac{\left(\varepsilon_{\mathrm{s}}-\varepsilon_{\mathrm{m}}\right)\left(\varepsilon+2 \varepsilon_{\mathrm{s}}\right)+g^{3}\left(2 \varepsilon_{\mathrm{s}}+\varepsilon_{\mathrm{m}}\right)\left(\varepsilon-\varepsilon_{\mathrm{s}}\right)}{\left(\varepsilon_{\mathrm{s}}+2 \varepsilon_{\mathrm{m}}\right)\left(\varepsilon+2 \varepsilon_{\mathrm{s}}\right)+g^{3}\left(2 \varepsilon_{\mathrm{s}}-2 \varepsilon_{\mathrm{m}}\right)\left(\varepsilon-\varepsilon_{\mathrm{s}}\right)}\right)
$$

where $g$ is the ratio of the diameter of the metal to the diameter of the total coated particle.

The absorption coefficient $K$ of the film can be written as

$$
K=\frac{2 \pi \lambda}{c^{2}} \frac{\operatorname{Im}\left(\varepsilon_{\text {eff }}\right)}{n_{\text {eff }}}
$$

where $\lambda$ is the wavelength of light, $c$ speed of light, and $n_{\text {eff }}$ is the real part of the effective complex index of refraction of the composite material, which is related to $\varepsilon_{\text {eff }}$ as:

$$
\left(n_{\text {eff }}+\mathrm{i} k_{\text {eff }}\right)^{2}=\varepsilon_{\text {eff }}
$$

and $k_{\text {eff }}$ is the imaginary part of the effective complex index of refraction.

Although a more complete description will be given elsewhere [25], we can see here from equations 2 and 5, that the peak position of the absorption band is predicted to red-shift with increasing volume fraction of metal particles, as well as with $\varepsilon_{\mathrm{m}}$ (refractive index of the surrounding medium). This means that the coupling can be controlled through the silica shell thickness determining interparticle separation, and therefore volume fraction.

We should finally mention that similar results concerning the screening of magnetic interactions were recently published for thin films of silica-coated magnetite nanoparticles [26].

\section{CONCENTRATED DISPERSIONS}

In this section, we also show how the optical properties of metal and semiconductor nanoparticles are affected by 3-dimensional nanostructuring with much larger interparticle distances. The first approach was based on the phase behavior of charged colloids. More specifically, we followed the work of Dhont et al [27] describing the phase behavior of silica particles derivatized with 3-(trimethoxysilyl)propyl methacrylate (TPM). The TPM-coated particles (which we denote $\mathrm{Au} @ \mathrm{SiO}_{2}$-TPM) are slightly charged due to the presence of residual hydroxide ions on the silica surface [28], and the sols are stable in solvents with a low polarity, such as ethanol and toluene, as well as in ethanol-toluene mixtures. For alcosols of these particles, the fluid-solid phase transition was observed to occur at a particle volume fraction of 0.194 , and the crystal-glass transition at 0.224 [27].

(C) 2000 IUPAC, Pure and Applied Chemistry 72, 257-267 

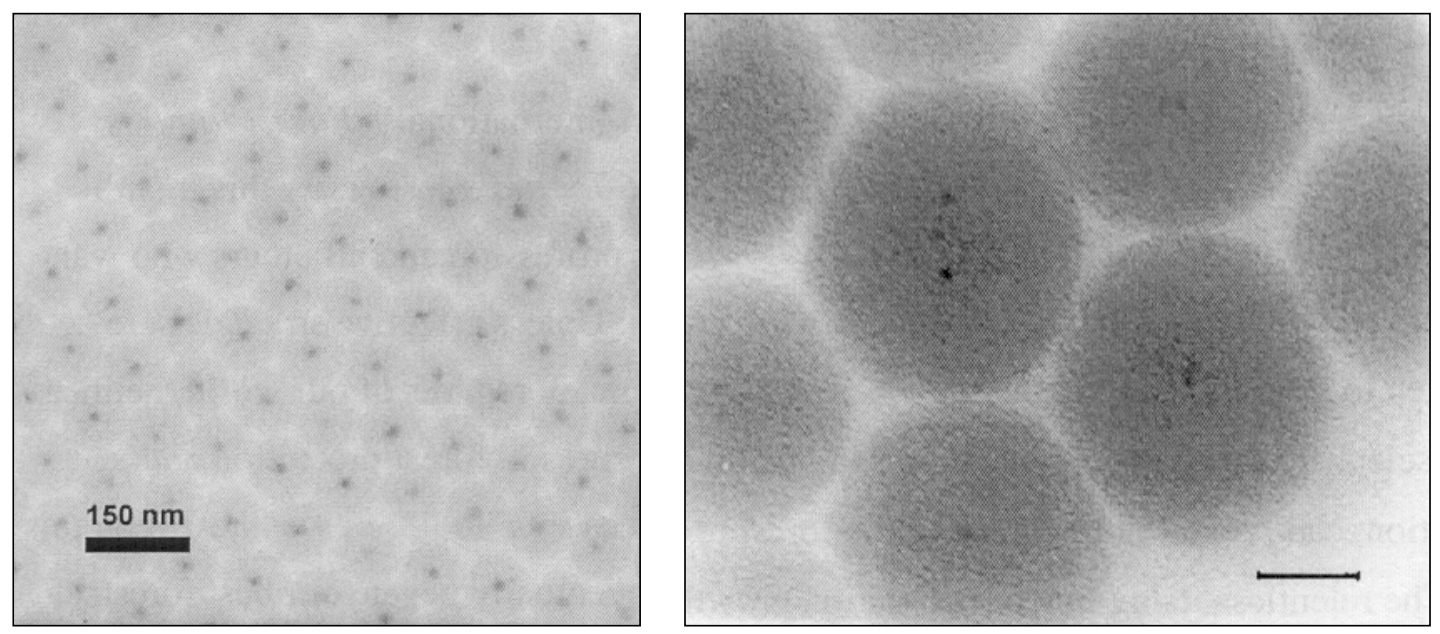

Fig. 4 Transmission electron micrographs of thickly coated Au (left) and CdS (right) nanoparticles.

Fig. 5 See color figure on page 265 .

We performed this study with both $\mathrm{Au} @ \mathrm{SiO}_{2}$ and $\mathrm{CdS} @ \mathrm{SiO}_{2}$, grown to final particle sizes of the order of $100 \mathrm{~nm}$, and derivatized with TPM as described in ref. 28. Transmission electron micrographs of these samples are shown in Fig. 4.

Concentrated dispersions of these modified core-shell particles were prepared by centrifugation inside glass cuvettes (1-mm path length) and dilution with pure ethanol, thus adjusting the particle volume fraction from 15 up to about 45 volume $\%$. In the case of gold cores, the effect of interparticle distance (volume fraction) can be easily distinguished by eye, as shown in Fig. 5 on page 265. The first cuvette (A) shows a concentration gradient obtained after centrifugation [29], while the other cuvettes contain the same particles with volume fractions increasing from left to right. It is obvious how the interplay between absorption by the cores and scattering by the shells leads to strong optical effects.

While the fluid phase (Fig. 5B, page 265) was turbid reddish-brown, the crystalline phase (Fig. $5 \mathrm{C}$, page 265) was bright red, showing Bragg diffraction in the form of brighter spots (iridescence) all over the sample. When the cuvette was tilted, a bright green color was observed, showing the presence of different lattice spacings for different diffraction angles. In the amorphous part of the phase diagram, the sample displayed different colors ranging from dark yellow to blue through green (Fig. 5D-F, page 265). The colors shown in the figure are those resulting from direct reflection of incident light, while the sample looked dark red when observed in transmission, which indicates that the reflected color indeed arises as a result of absorption and diffraction.

In the case of CdS cores, the visual effect is not so strong, since its absorption coefficient is much lower than that of gold. However, even using 1-mm path length cuvettes, the absorbance was too high for study with a conventional spectrophotometer. Therefore, we measured the specular reflectance of the samples with different concentrations, which indicates how the position of the Bragg peak red-shifts when decreasing volume fraction. This agrees with Bragg's law, when one considers both the change in filling factor when changing the volume fraction, and the increase in the particle refractive index due to the presence of the semiconductor cores. Assuming an fcc structure, the maximum filling factor is 0.74 . When there is open space between neighboring spheres (as in our case), the filling factor will actually be the particle volume fraction $(V)$. Therefore, the wavelength of the radiation diffracted by the crystal 
will depend on the filling factor through two terms. The lattice parameter, $\phi=0.816 \cdot d+a(V)$ depends on $V$ through $a$, the distance separating the spheres:

$$
a(V)=\frac{d\left[1-\left(\frac{V}{0.74}\right)^{\frac{1}{3}}\right]}{\left(\frac{V}{0.74}\right)^{\frac{1}{3}}}
$$

where $d$ is the diameter of the coated particle. On the other hand, the average dielectric constant is

$$
\varepsilon_{\mathrm{ef}}=V \cdot\left(\varepsilon_{\mathrm{silica}} \cdot(1-\eta)+\eta \cdot \varepsilon_{\mathrm{CdS}}\right)+(1-V) \cdot \varepsilon_{\mathrm{m}},
$$

where $\eta=\frac{R^{3}}{(d / 2)^{3}-R^{3}}$ is the ratio between the volumes of $\mathrm{CdS}$ and silica $(R=$ radius of $\mathrm{CdS}$ core $)$, and $\varepsilon_{\mathrm{m}}$ is the dielectric constant of the solvent.

Bragg's law $\lambda=2 \cdot \phi \cdot \sqrt{\varepsilon_{\mathrm{ef}}}$ then becomes:

$$
\lambda(V)=2 \cdot 0.816 \cdot d\left[1+\frac{\left[1-\left(\frac{V}{\theta .74}\right)^{\frac{1}{3}}\right.}{\left(\frac{V}{\delta_{0.74}}\right)^{\frac{1}{3}}}\right] \cdot \sqrt{\left[V \cdot\left[1.45^{2}\left(1-\frac{R^{3}}{\left.d^{3}\right)^{-}-R^{3}}\right]+2.56^{2} \frac{R^{3}}{\left.d^{\frac{d}{(}}\right)^{-}-R^{3}}\right]+(1-V) \cdot 1.33^{2}\right]}
$$

We show in Fig. 6 the specular reflectance spectra of dispersions of CdS@ $\mathrm{SiO}_{2}$ in ethanol for volume fractions between 18 and 30\%. As expected, the Bragg peak blue-shifts for increasing volume fractions, since the interparticle distance decreases accordingly. The inset shows a fit of the maximum position with eq. 8 .

\section{OPALS}

The final example that we are showing here of nanostructured systems based on core-shell nanoparticles is the preparation of opals, or in other words, solid crystalline arrangements of nanoparticles. The interest in opals has grown in the last few years since it implies a 3D modulation of the dielectric constant in a range well within the visible region of the electromagnetic spectrum, which can lead to forbidden bands in the transmission spectrum due to multiple Bragg diffraction. This photonic crystal behavior postulated by E. Yablonovich and S. John a decade ago [30,31] has opened up a new field in materials science, which is now starting to be exploited.

Several synthetic procedures have been proposed for the preparation of opals [32-35]. In this work, we have prepared opals from $\mathrm{CdS} @ \mathrm{SiO}_{2}$ and $\mathrm{Au} @ \mathrm{SiO}_{2}$ particles using the natural sedimentation method, developed by Meseguer et al [34,36]. The method simply consists of redispersing the particles in distilled water with a concentration of $1 \mathrm{wt} \%$, and allowing them to sediment, following Stokes law, which results in the formation of large, face-centered-cubic ordered compacts. The sphere diameter can be estimated from the sedimentation velocity, and making use of Stoke's law. For instance, in the case of 


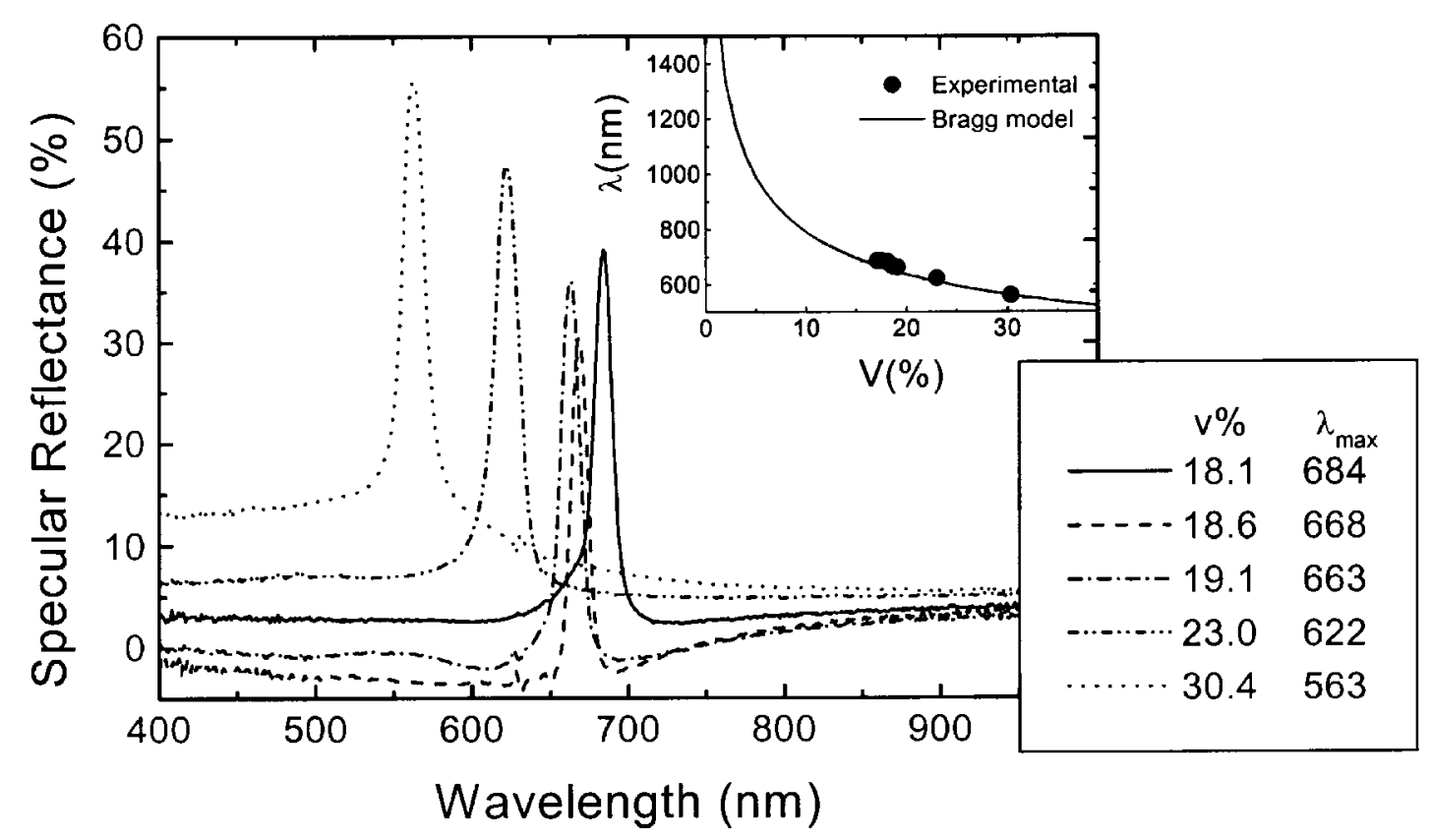

Fig. 6 Specular reflectance spectra of concentrated dispersions of $\mathrm{CdS} @ \mathrm{SiO}_{2}$ particles in ethanol. The inset shows a fit of the maximum position with eq. 8 .

the CdS@ $\mathrm{SiO}_{2}$ particles, a value of $224 \mathrm{~nm}$ was obtained, in good agreement with the value of $235 \mathrm{~nm}$ measured from SEM. Once the sedimentation has been completed, the solid is separated from the supernatant and dried at $60^{\circ} \mathrm{C}$. Examples of the ordered samples obtained by this method are shown in Fig. 7.

Although a more complete characterization of these systems is still to be performed, we present here some preliminary results on their photonic properties. Specular reflectance spectra of both Au and $\mathrm{CdS}$ opal samples are shown in Fig. 8. As expected from Bragg's law, the characteristic Bragg peak blueshifts as the incident angle increases. This effect has been summarized in the inset of Fig. 8 .

A fuller investigation of the photonic crystal properties exhibited by this type of material is justified by the large number of possible applications. For example, Au@ $\mathrm{SiO}_{2}$ opals can be used as metallodielectric photonic structures, which eventually show large photonic bandgaps when ordered in an fcc structure [37]. On the other hand, one of the most important consequences of photonic bandgaps in solids is the possibility to control spontaneous emission. If spontaneous emission is fully inhibited, high-efficiency lasers can be constructed. In particular, for CdS-doped silica opal composites, partial inhibition of the spontaneous emission has been recently shown [38]. With the core-shell structure used here, $\mathrm{CdS} @ \mathrm{SiO}_{2}$ opals can represent excellent systems to achieve spontaneous emission inhibition without photo-darkening effects.

\section{CONCLUSIONS}

We have demonstrated in this paper the numerous possibilities that silica-coated nanoparticle systems offer for the preparation of nanostructured materials with controlled optical properties. The enhanced stability provided by the silica shell allows for an easy manipulation of the nanoparticle units, so that different methods can be used for the preparation of nanostructures. 


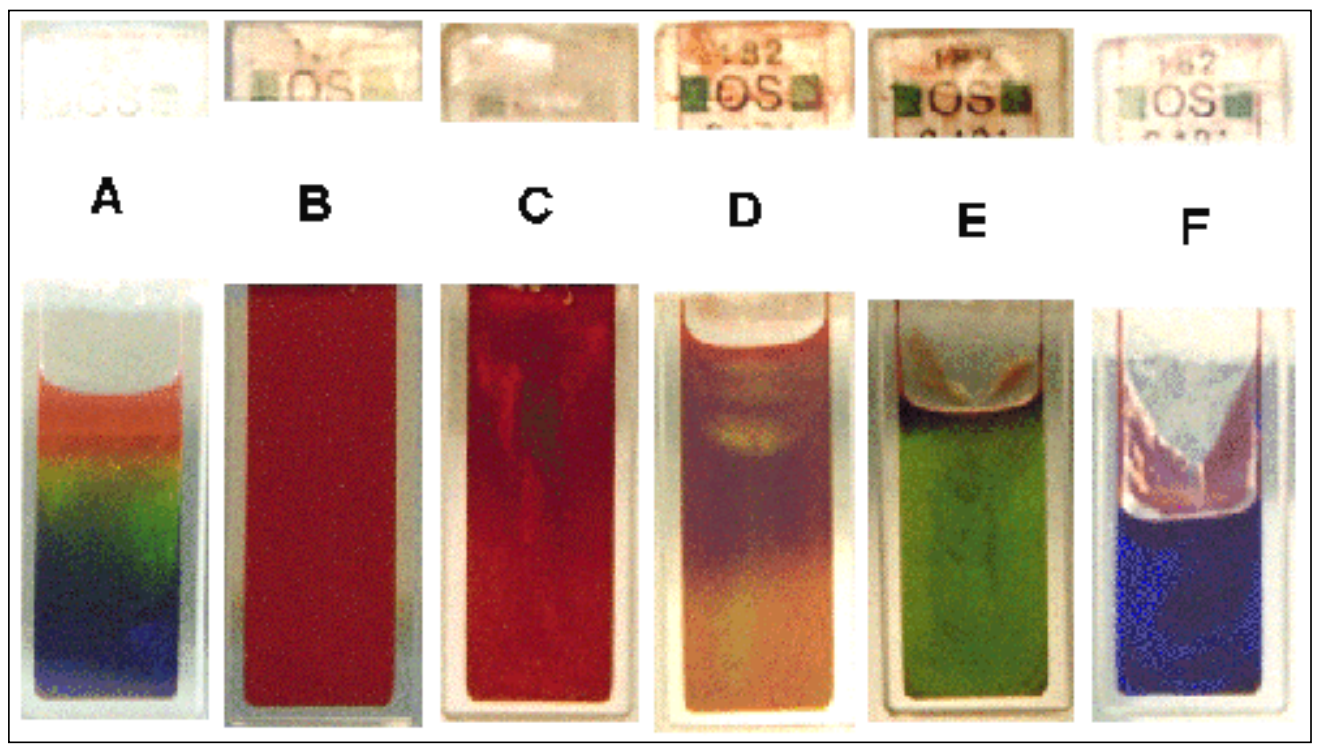

Fig. 5 Photographs of a dispersion of $\mathrm{Au} @ \mathrm{SiO}_{2}$ particles (core diameter: $15 \mathrm{~nm}$; total diameter: $105 \mathrm{~nm}$ ) in ethanol after centrifugation for $9 \mathrm{~h}$ and rest for 4 days (A); and concentrated dispersions of the same $\mathrm{Au} @ \mathrm{SiO}_{2}$ particles with increasing volume fractions: $16.7 \%$ (B); $20.2 \%$ (C); $27.8 \%$ (D); $35.1 \%$ (E); $54.6 \%$ (F).
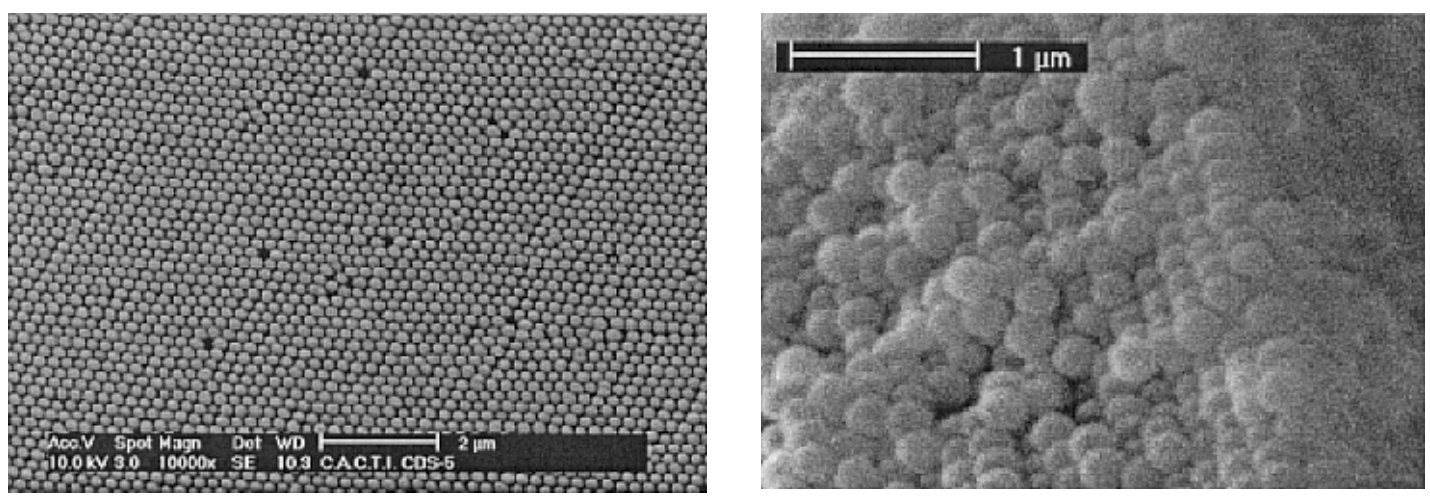

Fig. 7 Scanning electron micrographs showing the ordered structure of opals prepared from $\mathrm{CdS} @ \mathrm{SiO}_{2}(\mathrm{left})$ and $\mathrm{Au} @ \mathrm{SiO}_{2}$ (right) core-shell nanoparticles.

We have shown that thin films of $\mathrm{Au} @ \mathrm{SiO}_{2}$ can display a full range of colors, depending on interparticle separation. In this case, such separation is directly determined by the thickness of the silica shell deposited around each Au core. This optical effect has been explained on the basis of the screening of dipole-dipole interactions, which is confirmed by the fact that the spectra of films with sufficiently large interparticle spacings (around $30 \mathrm{~nm}$ ) basically coincides with that of the starting aqueous sol.

In the case of thickly coated metal and semiconductor nanoparticles, we showed that two different paths can be followed to prepare ordered three-dimensional structures. Colloidal crystals can be prepared in solution, just by playing around with the phase behavior of the colloid, and solid opals can be 

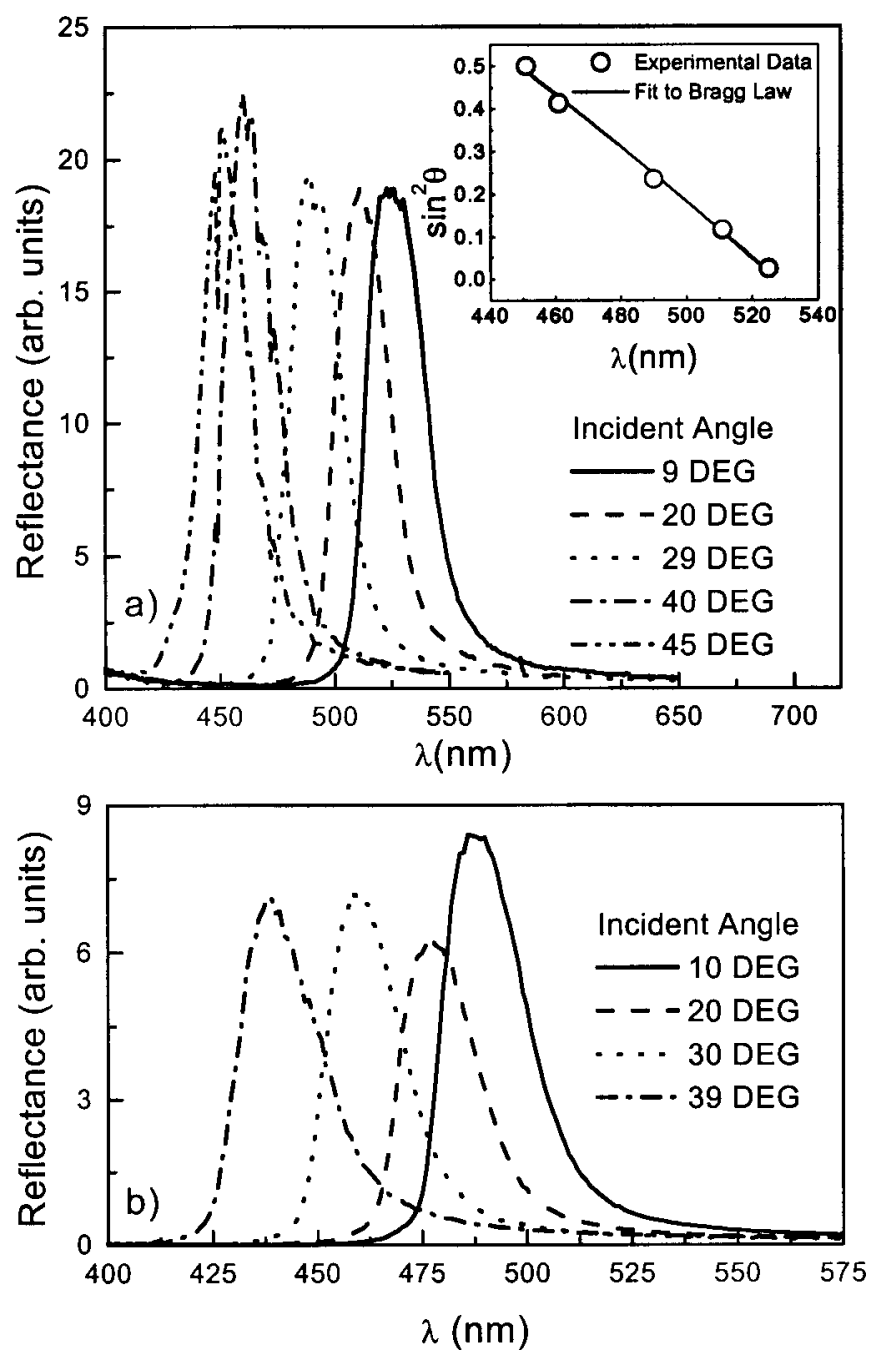

Fig. 8 Specular reflectance spectra measured from $\mathrm{CdS} @ \mathrm{SiO}_{2}$ (top) and $\mathrm{Au} @ \mathrm{SiO}_{2}$ (bottom) opals for different incident angles. The inset shows a fit of peak position vs. angle to Bragg's law.

synthesized, where once again the interparticle distance is accurately tuned by the thickness of the silica shell.

The nanostructuring of silica-coated nanoparticles is still to be fully developed, and promises a number of interesting applications in several fields of science and technology.

\section{ACKNOWLEDGMENTS}

Financial support by the Spanish Secretaría Xeral de Investigación e Desenvolvemento, Xunta de Galicia, Project no. PGIDT99PXI30104B is acknowledged. The C.A.C.T.I. from Vigo University is thanked for granting access to their microscopy facilities. The authors are grateful to J. B. Rodríguez for performing the SEM measurements.

\section{REFERENCES}

1. J. H. Fendler. Nanoparticles and Nanostructured Films, Wiley-VCH, Mainheim (1998).

2. A. Henglein. Chem. Rev. 89, 1861 (1989). 
3. G. Vigil, Z. Xu, S. Steinberg, J. Israelachvili. J. Colloid Interface Sci. 165, 367(1994).

4. R. K. Iler. U.S. Patent No. 2,885366 (1959).

5. M. Ohmori, E. Matijevic. J. Colloid Interface Sci. 150, 594 (1992).

6. A. P. Philipse, M. P. B. van Bruggen, C. Pathmamanoharan. Langmuir 10, 92 (1994).

7. A. P. Philipse, A. M. Nechifor, C. Pathmamanoharan. Langmuir 10, 4451 (1994).

8. L. M. Liz-Marzán, A. P. Philipse. J. Colloid Interface Sci. 176, 459 (1995).

9. S. Chang, L. Liu, S. A. Asher. J. Am. Chem. Soc. 116, 6739 (1994).

10. T. Li, J. Moon, A. Morrone, J. J. Mecholsky, D. R. Talham, J. H. Adair. Langmuir 15, 4328 (1999).

11. L. M. Liz-Marzán, M. Giersig, P. Mulvaney. J. Chem. Soc., Chem. Commun. 731 (1996).

12. L. M. Liz-Marzán, M. Giersig, P. Mulvaney. Langmuir 12, 4329 (1996).

13. E. P. Plueddermann. Silane Coupling Agents, 2nd ed., Plenum Press, New York (1991).

14. R. J. Puddephatt. The Chemistry of Gold, Elsevier, Amsterdam (1978).

15. M. A. Correa-Duarte, M. Giersig, L. M. Liz-Marzán.Chem. Phys. Lett. 286, 497 (1998).

16. W. Stöber, A. Fink, E. Bohn. J. Colloid Interface Sci. 26, 62 (1968).

17. R. J. Iler. J. Colloid Interface Sci. 21, 569 (1966).

18. Y. Lvov, G. Decher, H. Haas, H. Mohwald, A. Kalachev. Physica B 198, 89 (1994).

19. G. Decher. Science 277, 1232 (1997).

20. N. A. Kotov, I. Dekany, J. H. Fendler. J. Phys. Chem. 1995, 99, 13065-13069.

21. F. Caruso, H. Lichtenfeld, M. Giersig, H. Möhwald. J. Am. Chem. Soc. 1998, 120, 8523-8524.

22. M. A. Correa-Duarte, M. Giersig, N. A. Kotov, L. M. Liz-Marzán. Langmuir 14, 6430 (1998).

23. J. C. Maxwell-Garnett. Phil. Trans. Roy. Soc. (London) 203, 385 (1904); ibid, 205, 237 (1906).

24. D. A. Bruggemann. Ann. Phys. (Leipzig) 24, 634 (1935).

25. T. Ung, L. M. Liz-Marzán, P. Mulvaney, manuscript in preparation.

26. F. Aliev, M. A. Correa-Duarte, A. Mamedov, J. Ostrander, M. Giersig, L. M. Liz-Marzán, N. A. Kotov. Adv. Mater. 11, 1006 (1999).

27. J. K. G. Dhont, C. Smits, H. N. W. Lekkerkerker. J. Colloid Interface Sci. 152, 386 (1992).

28. A. P. Philipse and A. Vrij, J. Colloid Interface Sci. 128, 121 (1989).

29. J. S. van Duijneveldt, J. K. G. Dhont, H. N. W. Lekkerkerker. J. Chem. Phys. 99, 6941 (1993).

30. E. Yablonovitch Phys. Rev. Lett. 58, 2059 (1987); S. John, Ibid 2486.

31. J. D. Joannopoulos, R. D. Meade, J. N. Winn. Photonic Crystals, Princeton University Press, Princeton, NJ (1995).

32. O. D. Velev, T. A. Jede, R. F. Lobo, A. M. Lenhoff. Nature 389, 447 (1997).

33. A. van Blaaderen, R. Ruel, P. Wiltzius. Nature 385, 321 (1997).

34. R. Mayoral, J. Requena, S. J. Moya, C. López, A. Cintas, H. Míguez, F. Meseguer, L. Vázquez, M. Holgado, A. Blanco. Adv. Mater. 9, 257 (1997).

35. Yu. A. Vlasov, V. N. Astratov, O. Z. Karimov, A. A. Kaplyanskii, V. N. Bomogolov, A.V. Prokofiev. Phys. Rev. B 55, 13357 (1997).

36. H. Míguez, F. Meseguer, C. López, A. Blanco, J. S. Moya, J. Requena, A. Mifsud, V. Fornés. Adv. Mater. 10, 480 (1998).

37. E. R. Brown, O. B. McMahon. Appl. Phys. Lett. 67, 2138 (1995).

38. A. Blanco, C. López, R. Mayoral, H. Míguez, F. Meseguer, A. Mifsud, J. Herrero. Appl. Phys. Lett. 73, 1781 (1998).

(C) 2000 IUPAC, Pure and Applied Chemistry 72, 257-267 